\section{Riechvermögen bei allergischer Rhinitis gestört}

\author{
Riechstörungen können ein wichtiges Symptom bei Patienten mit \\ allergischer Rhinitis sein. Sie haben zum Teil deutliche Auswirkungen auf \\ die Lebensqualität der Betroffenen.
}

n einer systematischen Übersichtsarbeit zur entsprechenden Literatur hatten Wissenschaftler untersucht, wie genau der Einfluss einer allergischen Rhinitis auf den Geruchssinn ist und welche antiallergische Behandlung das Riechvermögen wiederherstellen könnte. Die Forscher fischten aus über 400 Arbeiten insgesamt 36 mit relevanten Daten für diese Fragestellung heraus.

Sie konnten zeigen, dass mit einer längeren Zeitdauer und einer stärkeren Ausprägung der allergischen Rhinitis auch die Häufigkeit von Geruchsstörungen zunimmt. Medikamentöse Therapien wie Antihistaminika und topische

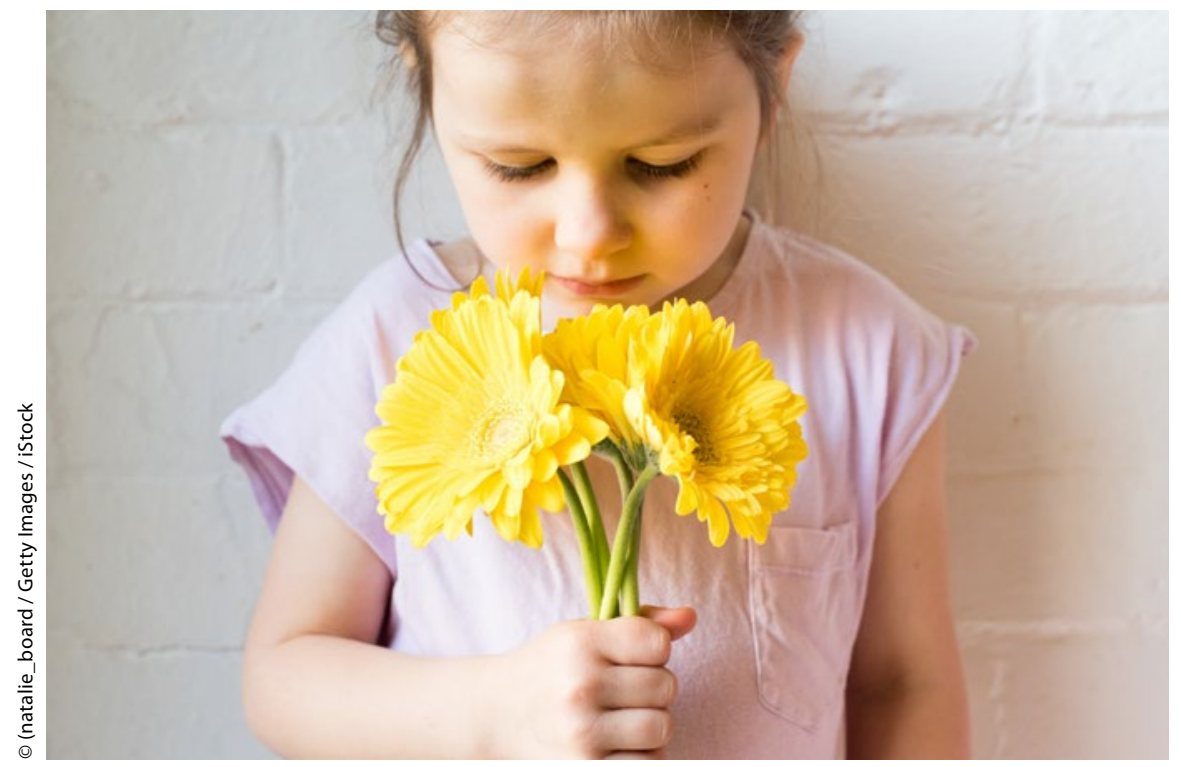

Bei Heuschnupfen können das Riechvermögen und damit auch die Lebensqualität stark eingeschränkt sein.

\section{Zusammenhang zwischen chronisch-spontaner Urtikaria und Parasiten}

Trotz ausführlicher diagnostischer Anstrengungen bleibt bei vielen Patienten mit Urtikaria die jeweilige Ursache ungeklärt. Eine deutsch-russische Übersichtsarbeit untersuchte nun eine mögliche Ätiologie durch Parasiten und Protozoen und klärte deren Häufigkeit sowie ihre möglichen Pathomechanismen ab.

U nter einer chronisch-spontanen Urtikaria versteht man eine persistierende Quaddelbildung oder Angioödeme (beziehungsweise auch eine Kombi-
Glukokortikoide konnten das Riechvermögen zum Teil nur mit begrenzter Evidenz verbessern. Ebenso war auch die spezifische Immuntherapie nur eingeschränkt von Nutzen, insbesondere wenn es sich um Patienten mit saisonaler allergischer Rhinitis handelte. Jedoch müssen viele Fragen teilweise ungelöst bleiben, weil kontrollierte und randomisierte Studien recht selten sind und nur ein geringer Teil der Studien eine aussagekräftige Messung der Riechfunktion aufweist.

Stuck BA et al. Olfaction in allergic rhinitis - a systematic review. J Allergy Clin Immunol 2015;136:1460-70

\section{Kommentar}

Patienten mit allergischer Rhinitis zeigen neben einer eingeschränkten Nasenatmung auch öfters eine Störung des Geruchsvermögens. Beides gemeinsam kann die Lebensqualität der Betroffenen deutlich einschränken. Insbesondere ist das Riechen bei länger anhaltender und stärker ausgeprägter allergischer Rhinitis beeinträchtigt. Antiallergische und antiinflammatorische Therapieansätze könnten hier positive Effekte erzielen - vor allem topische Glukokortikoide, die spezifische Immuntherapie und teilweise auch Antihistaminika. Für eine sinnvolle individuelle Behandlung sind weitere Studien erforderlich. Ebenso sollte auch das Riechvermögen bei den Patienten häufiger getestet werden, insbesondere da es hierfür bereits relativ einfache und auch kostengünstige Verfahren gibt.

Dr. Ulrich Mutschler

Ursachen einer Urtikaria wurden bereits mehrfach untersucht. In der vorliegenden Studie klassifizierten Allergologen aus Aachen, Berlin und Moskau nun die vorhandenen Veröffentlichungen näher und konnten 39 Studien zur Prävalenz einer Parasitenbesiedlung bei UrtikariaPatienten mit der chronisch-spontanen Form näher betrachten.

Bei etwa zwei Dritteln der Patienten fanden sich Infestationsraten von $10 \%$ oder weniger. Maximalwerte lagen bei einzelnen Studien zwischen 0 und $75 \%$ bei Erwachsenen und knapp $38 \%$ bei Kindern. Bei Patienten mit chronisch- 